\title{
Characteristics of new solid nodules detected in incidence screening rounds of low-dose CT lung cancer screening: the NELSON study
}

\author{
Joan E Walter, ${ }^{1}$ Marjolein A Heuvelmans, ${ }^{1,2}$ Geertruida H de Bock, ${ }^{3}$ \\ Uraujh Yousaf-Khan, ${ }^{4}$ Harry J M Groen, ${ }^{5}$ Carlijn M van der Aalst, ${ }^{4}$ Kristiaan Nackaerts, ${ }^{6}$ \\ Peter M A van Ooijen, ${ }^{1}$ Harry J de Koning, ${ }^{4}$ Rozemarijn Vliegenthart, ${ }^{1}$ \\ Matthijs Oudkerk'
}

\begin{abstract}
- Additional material is
published online only. To view please visit the journal online (http://dx.doi.org/10.1136/ thoraxjnl-2017-211376).

${ }^{1}$ Center for Medical Imaging - North East Netherlands, University of Groningen, University Medical Center Groningen, Groningen, The Netherlands

${ }^{2}$ Department of Pulmonology, Medisch Spectrum Twente Enschede, The Netherlands ${ }^{3}$ Department of Epidemiology, University of Groningen, University Medical Center Groningen, Groningen, The Netherlands

${ }^{4}$ Department of Public Health, Erasmus Medical Centre Rotterdam, Rotterdam, The Netherlands

${ }^{5}$ Department of Pulmonary

Diseases, University of Groningen, University Medical Center Groningen, Groningen, The Netherlands

${ }^{6}$ Department of Pulmonary Medicine, KU Leuven University Hospital Leuven, Leuven, Belgium
\end{abstract}

\section{Correspondence to}

Professor Matthijs Oudkerk, CMI-Center for Medical Imaging, University Medical Center Groningen, Groningen 9700RB, The Netherlands; m.oudkerk@umcg.nl

Received 5 December 2017 Revised 16 March 2018 Accepted 26 March 2018 Published Online First 16 April 2018

Check for updates

\begin{tabular}{l}
\hline To cite: Walter JE, \\
Heuvelmans MA, Bock GH, \\
et al. Thorax \\
2018;73:741-747.
\end{tabular}

\section{ABSTRACT}

Purpose New nodules after baseline are regularly found in low-dose CT lung cancer screening and have a high lung cancer probability. It is unknown whether morphological and location characteristics can improve new nodule risk stratification by size.

Methods Solid non-calcified nodules detected during incidence screening rounds of the randomised controlled Dutch-Belgian lung cancer screening (NELSON) trial and registered as new or previously below detection limit $\left(15 \mathrm{~mm}^{3}\right)$ were included. A multivariate logistic regression analysis with lung cancer as outcome was performed, including previously established volume cut-offs $\left(<30 \mathrm{~mm}^{3}, 30-<200 \mathrm{~mm}^{3}\right.$ and $\geq 200 \mathrm{~mm}^{3}$ ) and nodule characteristics (location, distribution, shape, margin and visibility $<15 \mathrm{~mm}^{3}$ in retrospect).

Results Overall, 1280 new nodules were included with $73(6 \%)$ being lung cancer. Of nodules $\geq 30 \mathrm{~mm}^{3}$ at detection and visible $<15 \mathrm{~mm}^{3}$ in retrospect, $22 \%$ (6/27) were lung cancer. Discrimination based on volume cut-offs (area under the receiver operating characteristic curve (AUC): $0.80,95 \% \mathrm{Cl} 0.75$ to 0.84 ) and continuous volume (AUC: $0.82,95 \% \mathrm{Cl} 0.77$ to 0.87 ) was similar. After adjustment for volume cut-offs, only location in the right upper lobe (OR 2.0, $\mathrm{P}=0.012)$, central distribution (OR 2.4, $P=0.001$ ) and visibility $<15 \mathrm{~mm}^{3}$ in retrospect (OR 4.7, $P=0.003$ ) remained significant predictors for lung cancer. The Hosmer-Lemeshow test $(P=0.75)$ and assessment of bootstrap calibration curves indicated adequate model fit. Discrimination based on the continuous model probability (AUC: $0.85,95 \% \mathrm{Cl} 0.81$ to 0.89 ) was superior to volume cut-offs alone, but when stratified into three risk groups (AUC: $0.82,95 \% \mathrm{Cl} 0.78$ to 0.86$)$, discrimination was similar.

Conclusion Contrary to morphological nodule characteristics, growth-independent characteristics may further improve volume-based new nodule lung cancer prediction, but in a three-category stratification approach, this is limited.

Trial registration number ISRCTN63545820; preresults.

\section{INTRODUCTION}

Lung cancer remains as a leading cause of cancer-related death worldwide, and US guidelines recommend lung cancer screening by low-dose CT

\section{Key messages}

What is the key question?

- Can morphological and location characteristics improve the currently endorsed size-based risk stratification of new nodules detected after baseline low-dose CT screening lung cancer screening?

What is the bottom line?

- In contrast to morphological characteristics, growth-independent characteristics such as location may further improve volume-based new nodule lung cancer prediction, but in the currently advocated stratification approach, this is limited.

Why read on?

- Management of new nodules determines the success of a lung cancer screening programme, and this is the first study to investigate the incremental value of morphological and location nodule characteristics to nodule volume cut-offs for lung cancer prediction in new nodules after baseline screening.

(LDCT) for high-risk individuals. ${ }^{1-5}$ Presently, lung cancer screening guidelines and nodule management protocols primarily focus on size and nodule growth for risk stratification, but the potential incremental value of morphological and location nodule characteristics has been underlined. ${ }^{6-11}$ It has been reported that nodules smaller than 5-6 mm (roughly $65-113 \mathrm{~mm}^{3}$ ) have a very low likelihood of being lung cancer. ${ }^{8} 101213$ However, current knowledge concerning nodule management in lung cancer screening is mainly based on baseline nodules that may have been present for years before their detection. ${ }^{14} 15$ New nodules after baseline develop within a known timeframe and entail a group of young and potentially fast-growing nodules. Recently, the Dutch-Belgian lung cancer screening trial (NELSON) published a more detailed analysis on new nodules detected in incidence screening rounds. ${ }^{14}$ It was shown that compared with baseline nodules, new solid nodules possess a greater lung cancer probability already at smaller 
size, ${ }^{13} 14$ and subsequent data from the National Lung Cancer Screening Trial indicated similar findings. ${ }^{16}$ Furthermore, it has been suggested that participants with new nodule lung cancer have poorer survival outcomes compared with participants who had at least one positive screen prior to cancer diagnosis. ${ }^{17}$ Based on the results in the NELSON trial, it was proposed in an European position statement on lung cancer screening that new solid nodules identified at an incidence screen and $<30 \mathrm{~mm}^{3}$ volume (adapted from $27 \mathrm{~mm}^{3},<1 \%$ lung cancer probability) or $<4 \mathrm{~mm}$ (simulated mean) diameter (adapted from $3.7 \mathrm{~mm},<1 \%$ lung cancer probability) comprise low risk nodules, new solid nodules $30-<200 \mathrm{~mm}^{3}$ (adapted from $206 \mathrm{~mm}^{3}$, around $3 \%$ lung cancer probability) or $4-8 \mathrm{~mm}$ (simulated mean) diameter (adapted from $8.2 \mathrm{~mm}$, around $3 \%$ lung cancer probability) represent indeterminate risk nodules and new solid nodules $\geq 200 \mathrm{~mm}^{3}$ (around 17\% lung cancer probability) or $\geq 8 \mathrm{~mm}$ (simulated mean) diameter (around 14\% lung cancer probability) are highrisk nodules, which was also adopted in the British Thoracic Society Guidelines for the Investigation and Management of Pulmonary Nodules. ${ }^{11} 14$

In the clinical setting, physicians evaluate solid pulmonary nodules based on their size and based on their morphological and location characteristics, and likewise in lung cancer screening, the expertise of a radiologist was shown to decrease false-positive screen results. ${ }^{9}{ }^{18} 19$ Nodule location in the upper lung and right upper lung in particular as well as marginal spiculation have been typically identified as risk factor for lung cancer in screening studies. ${ }^{10}{ }^{16} 20-22$ Nevertheless, consistent characterisation of very small nodules can be challenging and evidence concerning the discriminative value of new nodule morphology and location for lung cancer in new nodules in incidence screening rounds of LDCT screening is lacking.

The aim of this study was to assess whether addition of morphological and location characteristics to currently proposed volume-based three-category risk stratification can improve management of new solid nodules in LDCT lung cancer screening.

\section{METHODS}

\section{Study population}

The NELSON trial was authorised by the Dutch Health Care Committee. All participants provided written informed consent. The recruitment process and study design were published before. $^{72324}$ Summarised, eligible patients were adults aged $50-75$ years, who had smoked $>15$ cigarettes per day for $>25$ years or $>10$ cigarettes per day for $>30$ years and were still smoking or stopped smoking $<10$ years previously. Between April 2004 and December 2006, 7557 participants underwent baseline screening in four centres in the Netherlands and Belgium. The incidence screening rounds took place 1 year, 3 years and 5.5 years after baseline screening. In this study, participants with a solid non-calcified nodule detected during the incidence screening rounds and registered by the NELSON radiologists as new or $<15 \mathrm{~mm}^{3}$ (study detection limit) at previous screens were included.

\section{CT scanning protocol}

The CT protocol was published before. ${ }^{724}$ The four screening sites used 16-MDCT scanners or 64-MDCT scanners (Sensation-16, Siemens Medical Solutions, Forchheim, Germany, or Mx8000 IDT or Brilliance 16P, Philips Medical Systems, Best, The Netherlands). Scans of the entire chest were performed without contrast in spiral mode in caudio-cranial direction with $16 \times 0.75 \mathrm{~mm}$ collimation and 1.5 pitch. Low dose settings $(80-90 \mathrm{kVp}, 120 \mathrm{kVp}$ and $140 \mathrm{kVp})$ were adjusted depending on body weight $(<50 \mathrm{~kg}, 50-80 \mathrm{~kg}$ or $>80 \mathrm{~kg})$, matching a CT dose index volume of $0.8 \mathrm{mGy}, 1.6 \mathrm{mGy}$ or $3.2 \mathrm{mGy}$, respectively. Datasets were derived from images of the thorax with $1.0 \mathrm{~mm}$ slice width and a $0.7 \mathrm{~mm}$ reconstruction interval. Screening conditions and data acquisition were standard across screening sites. $^{724}$

\section{Image reading}

In the first two rounds, two independent radiologists with experience in thoracic CT reading ranging between 1 year and 20 years evaluated each CT scan individually, and in case of discrepancy, a third reader made the final decision. ${ }^{724}$ In the third and fourth screening rounds, single read was performed by radiologists with at least 6 years of experience in thoracic imaging after it was shown that double reading consensus has no benefit when using semiautomated software. ${ }^{25}$ CT data analysis was performed on digital workstations (Leonardo, Siemens Medical Solutions) using software for semiautomated volume measurements (LungCare, version Somaris/5 VA70C-W, Siemens Medical Solutions). Lung windows were assessed at a width of $1600 \mathrm{HU}$ and a level of $-700 \mathrm{HU}$. All images were interpreted both in lung window and mediastinal settings.

A non-calcified nodule was considered solid if the underlying structures were completely obscured by its lung opacity (8). A nodule's shape was classified as spherical, polygonal or irregular. ${ }^{19}$ The nodule margin was classified as smooth, lobulated, spiculated or irregular. ${ }^{21}{ }^{26}$ Additional to the nodule location in the lung, the distribution (peripheral and central) within the lung parenchyma was characterised based on the distance to the costal pleura. If the distance to costal pleura was less than one-third of the total distance to hilum-costal pleura, the nodule was defined as peripheral and if it was more than one-third, the nodule was defined as central. ${ }^{19} 27$ After detection, the software's matching algorithm matched nodules individually (depending on consistency, size and location) with previous scans and the radiologists visually confirmed the matching. In this analysis, a nodule was considered new if it was registered by the radiologist as new or below the study detection limit of $15 \mathrm{~mm}^{3}$ on the previous scan. Radiologists could overrule protocol-based screening result (done for 195 (6\%) of 3318 participants at the baseline screening round) in case of high suspicion of malignancy (eg, enlarged mediastinal lymph nodes) or benignity (eg, benign calcification patterns) and to adjust the volume measurement in case of inappropriate segmentation. ${ }^{18}$ Data obtained during CT evaluation were directly uploaded to the NELSON management system. ${ }^{7}$ For this study, nodule information as reported by the radiologists in the NELSON management system was used, and no retrospective measurements was performed.

\section{Nodule management protocol}

The NELSON nodule management protocol has been described in detail previously. ${ }^{7}$ Summarised, solid nodules detected within the NELSON trial were classified into four categories (NODCAT I-IV) according to their size and benign characteristics. After baseline screening, calcified nodules or nodules with other benign characteristics were considered benign (NODCAT I), new solid nodules without benign characteristics measuring $15-50 \mathrm{~mm}^{3}$ (NODCAT II, follow-up LDCT within 1 year) and new solid nodules $50-500 \mathrm{~mm}^{3}$ (NODCAT 
1,484 new solid nodules registered in 949 participants during the three incidence screening rounds

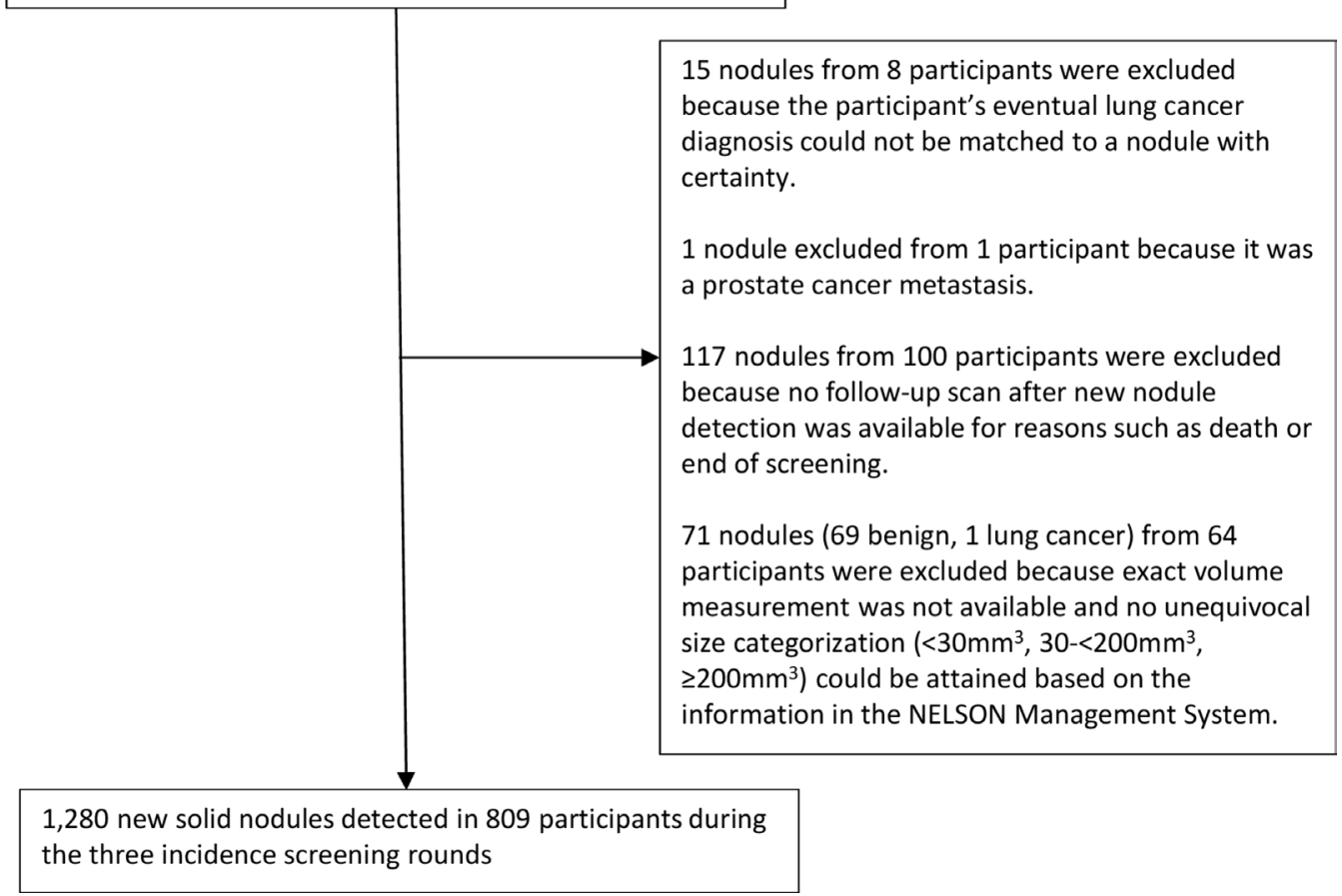

Figure 1 Flow chart of new solid nodules included in this analysis.

III, follow-up LDCT within 6-8 weeks) were defined indeterminate, whereas nodules measuring $\geq 500 \mathrm{~mm}^{3}$ (NODCAT IV, immediate referral to pulmonologist) were considered positive. After initial detection, a nodule's subsequent evaluation was based on volume doubling time. ${ }^{7}$ A volume doubling time $<400$ days led to referral to a pulmonologist for further diagnosis.

\section{Outcomes}

For this study, a nodule was classified as lung cancer when it was diagnosed as lung cancer during diagnostic workup according to national and international guidelines including histological examination. ${ }^{7}$ Nodules were classified as benign when either: (A) the nodule was benign at histological examination; (B) extensive diagnostic evaluation had a negative finding; $(\mathrm{C})$ the nodule was ruled negative during the participant's last follow-up screening of the NELSON trial and the participant did not present with postscreening lung cancer according to the Dutch and Belgian national cancer registries and medical file review. ${ }^{71328}$

\section{Previously established volume cut-offs for new nodules at initial detection}

Considering a previous analysis of the first two incidence screening rounds of the NELSON trial concerning optimal new nodule volume cut-offs at initial detection, ${ }^{14}$ nodules were classified as $<30 \mathrm{~mm}^{3}$ (low risk), $30-<200 \mathrm{~mm}^{3}$ (intermediate risk) or $\geq 200 \mathrm{~mm}^{3}$ (high risk) based on their semiautomated volume measurements (continuous volume) or the radiologist's nodule classification $\left(<50 \mathrm{~mm}^{3}\right.$, $50-500 \mathrm{~mm}^{3},>500 \mathrm{~mm}^{3}$; performed for $4 \%(50 / 1280)$ of the included nodules). ${ }^{11}$

\section{Statistical analysis}

The normality assumption was tested using the Kolmogorov-Smirnov test as well as visual assessment. Continuous variables are presented as median and IQR, and categorical variables are presented as frequencies and respective percentages. CIs of proportions were calculated using the Agresti-Coull method. The Mann-Whitney U test was used to compare the nodule volume of benign nodules and lung cancers at initial detection. Nominal variables were analysed with Fisher's exact test. Logistic regression analysis with new nodule lung cancer as outcome was performed to assess morphological and location nodule characteristics together with the previously established new nodule volume cut-offs $\left(<30 \mathrm{~mm}^{3}, 30-<200 \mathrm{~mm}^{3}\right.$ and $\left.\geq 200 \mathrm{~mm}^{311}{ }^{14}\right)$. The final parsimonious model included nodule characteristics that were significant $(\mathrm{P}<0.05)$ for new nodule lung cancer when adjusted for the volume cut-offs. The model calibration was assessed using the Hosmer-Lemeshow goodness-of-fit test and bootstrap calibration plots of actual probability versus predicted probability, with ideal, apparent and bias-corrected curves. The model probability was stratified through assessment of Youden Indices to maximise the area under the receiver operating characteristic curve (AUC) for three categories (termed low risk, intermediate risk and high risk) and provide at least $95 \%$ sensitivity, analogous to the previously established volume cut-offs. ${ }^{14}$ The performance for discriminating new nodule lung cancer was quantified by the AUC. The model performance was internally validated using 10 -fold cross-validation. AUC comparison was performed with the method described by DeLong et al. ${ }^{29}$ Decision curve analysis was used to estimate clinical usefulness of the model by plotting the net benefit (y-axis) over a continuum of potential decision probability thresholds (x-axis). ${ }^{3031}$ The net benefit represents the sum of true-positive minus false-positive classifications 
Table 1 Frequency of nodule characteristics stratified by lung cancer diagnosis and size

\begin{tabular}{|c|c|c|c|c|c|c|c|}
\hline & & & cancer & & Proportion of & with characteri & ng lung cancer \\
\hline & $\begin{array}{l}\text { Overall, } \\
100 \%(n=1280)\end{array}$ & $\begin{array}{l}\text { No, } \\
94 \%(n=1207)\end{array}$ & $\begin{array}{l}\text { Yes, } \\
6 \%(n=73)\end{array}$ & $P$ values & $\begin{array}{l}<30 \mathrm{~mm}^{3}, \\
33 \%(\mathrm{n}=429)\end{array}$ & $\begin{array}{l}30-<200 \mathrm{~mm}^{3}, \\
43 \%(\mathrm{n}=547)\end{array}$ & $\begin{array}{l}\geq 200 \mathrm{~mm}^{3}, \\
24 \%(\mathrm{n}=304)\end{array}$ \\
\hline Nodule volume & & & & & $0.5(2 / 429)$ & $3(18 / 547)$ & $17(53 / 304)$ \\
\hline$<30 \mathrm{~mm}^{3}$ & $34(429 / 1280)$ & $35(427 / 1207)$ & $3(2 / 73)$ & $<0.0001$ & - & - & - \\
\hline $30-<200 \mathrm{~mm}^{3}$ & $43(547 / 1280)$ & $44(529 / 1207)$ & $25(18 / 73)$ & 0.001 & - & - & - \\
\hline$\geq 200 \mathrm{~mm}^{3}$ & $24(304 / 1280)$ & $21(251 / 1207)$ & $73(53 / 73)$ & $<0.0001$ & - & - & - \\
\hline Median $\left(\mathrm{mm}^{3}\right)$ (IQR) & $50(23-156)$ & $47(22-132)$ & $387(124-1017)$ & $<0.0001$ & - & - & - \\
\hline Location & & & & & & & \\
\hline Right upper lung & $26(327 / 1277)$ & $25(299 / 1204)$ & $38(28 / 73)$ & 0.013 & $2(2 / 100)$ & $5(7 / 155)$ & $26(19 / 72)$ \\
\hline Left upper lung & $21(273 / 1277)$ & $22(259 / 1204)$ & $19(14 / 73)$ & 0.769 & $0(0 / 94)$ & $4(5 / 122)$ & $16(9 / 57)$ \\
\hline Right lower lung & $33(424 / 1277)$ & $34(207 / 1204)$ & $23(17 / 73)$ & 0.073 & $0(0 / 143)$ & $1(2 / 166)$ & $13(15 / 115)$ \\
\hline Left lower lung & $20(253 / 1277)$ & $20(239 / 1204)$ & $19(14 / 73)$ & 1.000 & $0(0 / 92)$ & $4(4 / 103)$ & $17(10 / 58)$ \\
\hline Right or left lung & & & & & & & \\
\hline Right lung & $59(751 / 1277)$ & $59(706 / 1204)$ & $62(45 / 73)$ & 0.714 & $1(2 / 243)$ & $3(9 / 321)$ & $18(34 / 187)$ \\
\hline Left lung & $41(526 / 1277)$ & $41(498 / 1204)$ & $38(28 / 73)$ & 0.714 & $0(0 / 186)$ & $4(9 / 225)$ & $17(19 / 115)$ \\
\hline Distribution & & & & & & & \\
\hline Central & $22(281 / 1272)$ & $21(255 / 1199)$ & $36(26 / 73)$ & 0.008 & $0(0 / 87)$ & $6(8 / 134)$ & $30(18 / 60)$ \\
\hline Peripheral & $78(991 / 1272)$ & 79 (944/1199) & $64(47 / 73)$ & 0.008 & $1(2 / 341)$ & $2(10 / 410)$ & $15(35 / 240)$ \\
\hline Shape & & & & & & & \\
\hline Round & $56(638 / 1137)$ & $57(613 / 1076)$ & $41(25 / 61)$ & 0.017 & $0.3(1 / 311)$ & $2(4 / 255)$ & $28(20 / 72)$ \\
\hline Polygonal & $35(400 / 1137)$ & 35 (378/1076) & $36(22 / 61)$ & 0.891 & $1(1 / 94)$ & $4(8 / 204)$ & $13(13 / 102)$ \\
\hline Irregular & $9(99 / 1137)$ & $8(85 / 1076)$ & $23(14 / 61)$ & 0.0004 & $0(0 / 2)$ & $5(1 / 20)$ & $17(13 / 77)$ \\
\hline Margin & & & & & & & \\
\hline Smooth & $53(671 / 1260)$ & $55(656 / 1189)$ & $21(15 / 71)$ & $<0.0001$ & $0.3(1 / 390)$ & $3(7 / 244)$ & $19(7 / 37)$ \\
\hline Lobulated & $36(453 / 1260)$ & $35(417 / 1189)$ & $51(36 / 71)$ & 0.010 & $0(0 / 32)$ & $4(10 / 272)$ & $17(26 / 149)$ \\
\hline Spiculated & $7(82 / 1260)$ & $6(68 / 1189)$ & $20(14 / 71)$ & $<0.0001$ & $0(0 / 2)$ & $0(0 / 12)$ & $21(14 / 68)$ \\
\hline Irregular & $4(54 / 1260)$ & $4(48 / 1189)$ & $9(6 / 71)$ & 0.119 & $33(1 / 3)$ & $0(0 / 12)$ & $13(5 / 39)$ \\
\hline Visibility in retrospect & & & & & & & \\
\hline Not visible & $80(1025 / 1280)$ & $80(960 / 1207)$ & $89(65 / 73)$ & 0.050 & $0(0 / 201)$ & $3(14 / 528)$ & $17(51 / 296)$ \\
\hline Small nodule $<15 \mathrm{~mm}^{3}$ & $20(255 / 1280)$ & $20(247 / 1207)$ & $11(8 / 73)$ & 0.050 & $1(2 / 228)$ & $21(4 / 19)$ & $25(2 / 8)$ \\
\hline
\end{tabular}

Missing values were excluded from analyses.

weighted by the respective probability threshold (eg, a decision threshold of $10 \%$ would imply that for every true-positive classification nine false-positive classifications are clinically acceptable). Missing data were excluded from the respective analyses. Statistical analyses were performed with SPSS V.25.0 (IBM, Armonk, USA), R V.3.3.3 (R Foundation for Statistical Computing, Vienna, Austria) and Microsoft Excel 2010 (Microsoft Corporation, Redmond, Washington, USA).

\section{RESULTS}

In total, 1280 new solid nodules detected in 809 participants during the three incidence rounds were included (figure 1). Median participant age at baseline screening was 59 years (IQR 55-63 years), and 77\% (622/809) were male. Of the included nodules, 20\% (255/1280) were visible as small nodule $<15 \mathrm{~mm}^{3}$ in retrospect.

Eventually, 6\% (73/1280) of the new solid nodules were diagnosed as lung cancer. At initial detection, median nodule volume was $50 \mathrm{~mm}^{3}$ (IQR 23-156 $\mathrm{mm}^{3}$ ) with $34 \%(429 / 1280)$ being $<30 \mathrm{~mm}^{3}, 43 \%(547 / 1280)$ being $30-<200 \mathrm{~mm}^{3}$ and $24 \%$ (304/1280) being $\geq 200 \mathrm{~mm}^{3}$.
Table 1 presents the nodule characteristics of benign new solid nodules and lung cancers at initial nodule detection and the proportion of lung cancers stratified by the volume cut-offs.

Overall, new solid nodules visible in retrospect $<15 \mathrm{~mm}^{3}$ were smaller and less often lung cancer compared with new solid nodules not visible in retrospect $(3 \%(8 / 255)$ vs $6 \%(65 / 1025))$. However, of new solid nodules $\geq 30 \mathrm{~mm}^{3}$ at detection and visible $<15 \mathrm{~mm}^{3}$ in retrospect, $22 \%(6 / 27)$ were lung cancer compared with $8 \%(65 / 824)$ of new solid nodules $\geq 30 \mathrm{~mm}^{3}$ and not visible in retrospect.

Table 2 displays the results of the logistic regression analysis.

Larger volume, location in the right upper lung, central distribution, irregular shape and a lobulated or spiculated margin were associated with lung cancer in univariate analysis. After addition of the volume cut-off categories to the selected nodule characteristics by multivariate logistic regression, only location in the right upper lung and central distribution significantly improved lung cancer prediction. Furthermore, after addition of the volume cut-offs, visibility in retrospect as small nodule $<15 \mathrm{~mm}^{3}$ was significantly associated with lung cancer. In other words, at equivalent size a new solid nodule visible in retrospect $<15 \mathrm{~mm}^{3}$ was more likely lung cancer than a new nodule not visible at all. 
Table 2 Logistic regression analysis of nodule characteristics and volume cut-offs with lung cancer as outcome

\begin{tabular}{|c|c|c|c|c|c|c|c|}
\hline & \multicolumn{2}{|l|}{ Univariate analysis } & \multicolumn{2}{|c|}{$\begin{array}{l}\text { Volume cut-offs added to each } \\
\text { characteristic }\end{array}$} & \multicolumn{3}{|l|}{ Full model } \\
\hline & OR $(95 \% \mathrm{Cl})$ & $P$ values & OR $(95 \% \mathrm{Cl})$ & $P$ values & OR $(95 \% \mathrm{Cl})$ & $P$ values & Beta coefficient \\
\hline \multicolumn{8}{|l|}{ Volume cut-off values } \\
\hline$<30 \mathrm{~mm}^{3}$ & Reference & & & & Reference & & \\
\hline $30-<200 \mathrm{~mm}^{3}$ & 7.3 (1.7 to 31.5$)$ & 0.008 & & & $16.8(3.2$ to 85.4$)$ & 0.001 & 2.818 \\
\hline$\geq 200 \mathrm{~mm}^{3}$ & 45.1 (10.9 to 186.6) & $<0.0001$ & & & 128.1 (25.1 to 651.9$)$ & $<0.0001$ & 4.852 \\
\hline \multicolumn{8}{|l|}{ Location } \\
\hline Right upper lung & 1.9 (1.2 to 3.1$)$ & 0.011 & 2.1 (1.3 to 3.5$)$ & 0.005 & $2.0(1.2$ to 3.4$)$ & 0.012 & 0.687 \\
\hline Not right upper lung & Reference & & Reference & & Reference & & \\
\hline \multicolumn{8}{|l|}{ Right or left lung } \\
\hline Right lung & 1.1 (0.7 to 1.8$)$ & 0.613 & 1.0 (0.6 to 1.7$)$ & 0.904 & & & \\
\hline Left lung & Reference & & Reference & & & & \\
\hline \multicolumn{8}{|l|}{ Distribution } \\
\hline Central & 2.0 (1.2 to 3.4$)$ & 0.005 & 2.4 (1.4 to 4.1$)$ & 0.001 & 2.4 (1.4 to 4.2 ) & 0.001 & 0.885 \\
\hline Peripheral & Reference & & Reference & & Reference & & \\
\hline \multicolumn{8}{|l|}{ Shape } \\
\hline Spherical & Reference & & Reference & & & & \\
\hline Polygonal & 1.4 (0.8 to 2.6$)$ & 0.235 & 0.7 (0.4 to 1.4$)$ & 0.327 & & & \\
\hline Irregular & 4.0 (2.0 to 8.1$)$ & $<0.0001$ & 0.8 (0.4 to 1.7$)$ & 0.512 & & & \\
\hline \multicolumn{8}{|l|}{ Margin } \\
\hline Smooth & Reference & & Reference & & & & \\
\hline Lobulated & 3.8 (2.0 to 7.0$)$ & $<0.0001$ & 1.1 (0.6 to 2.2 ) & 0.722 & & & \\
\hline Spiculated & 9.0 (4.2 to 19.4$)$ & $<0.0001$ & 1.3 (0.5 to 3.1$)$ & 0.576 & & & \\
\hline Irregular & 5.4 (2.0 to 14.7$)$ & 0.001 & 0.8 (0.3 to 2.6$)$ & 0.880 & & & \\
\hline \multicolumn{8}{|l|}{ Visibility in retrospect } \\
\hline Not visible & Reference & & Reference & & Reference & & \\
\hline Small nodule $<15 \mathrm{~mm}^{3}$ & $0.5(0.2$ to 1.0$)$ & 0.053 & $4.7(1.8$ to 12.7$)$ & 0.002 & 4.7 (1.7 to 12.8$)$ & 0.003 & 1.543 \\
\hline
\end{tabular}

Missing values were excluded from analyses. Full model equation: logit(p) $=-5.31+$ volume $30-<200 \mathrm{~mm}^{3 *} 2.818+$ volume $>200 \mathrm{~mm}^{3 *} 4.825+$ location in right upper

lung ${ }^{\star} 0.687+$ central distribution ${ }^{*} 0.885+$ visibility in retrospect as small nodule $<15 \mathrm{~mm}^{3 *} 1.543$.

The full model included the nodule volume cut-offs, location in the lung, distribution in the lung and visibility in retrospect.

Figure 2 displays the ROC curves of the volume cut-off values, the full model and the model stratified into three risk categories. Discrimination based on volume cut-offs (AUC: $0.80,95 \% \mathrm{CI}$ $0.75-0.84$ ) and continuous volume (AUC: $0.82,95 \%$ CI 0.77 to 0.87 ) was similar. The full model (AUC: $0.85,95 \%$ CI 0.81 to 0.89 ) provided superior discriminative performance compared with the volume cut-offs alone. The 10 -fold cross-validated mean AUC was similar $(0.846 \pm 0.050)$. The Hosmer-Lemeshow test was non-significant $(\mathrm{P}=0.75)$ suggesting a good overall fit (online supplementary figure 1 ). The clinical utility of the model in terms of an increased number of true positive predictions without increase in the false-positive rate (net benefit) was assessed over a continuum of potential risk thresholds using decision curve analysis (figure 3). The model displayed consistent positive and larger net benefit for risk thresholds above 2\% (intermediate and high-risk nodule thresholds) when compared with the volume cut-offs alone. Nevertheless, when stratifying nodules into three categories based on model cut-off values for a maximal AUC (AUC: $0.82,95 \% \mathrm{CI} 0.78$ to 0.86 ), thereby reflecting a three risk-group stratification (low risk, intermediate risk and high risk), there was no significant difference in discriminatory performance compared with the volume cut-offs.

\section{DISCUSSION}

This study aimed to evaluate the potential incremental value of morphological and location characteristics to volume-based lung cancer risk stratification of new solid nodules identified after baseline LDCT lung cancer screening. Overall, 1280 new solid nodules detected in 809 participants during the incidence screening rounds of the NELSON trial were included, with 6\% (73/1280) being diagnosed as lung cancer. Studies on new nodules detected in incidence screening rounds of LDCT screening are sparse, and only limited evidence concerning management of these nodules exist. ${ }^{81432}$ To our knowledge, this is the first study to investigate the incremental value of morphological and location nodule characteristics to nodule volume cut-offs for lung cancer prediction in new nodules after baseline screening.

We report five central findings. First, at initial detection new solid nodule volume and, therefore, its growth speed was the strongest predictor for malignancy. Second, nodule features traditionally attributed to lung cancer, such as location in the upper lung, central distribution, irregular shape and a lobulated or spiculated margin, were associated with lung cancer in new solid nodules in univariate logistic regression analysis. This is consistent with previous findings, mainly in baseline nodules. ${ }^{16-21}$ Third, when added to the previously established new solid nodule volume cut-off values $\left(<30 \mathrm{~mm}^{3}\right.$, 


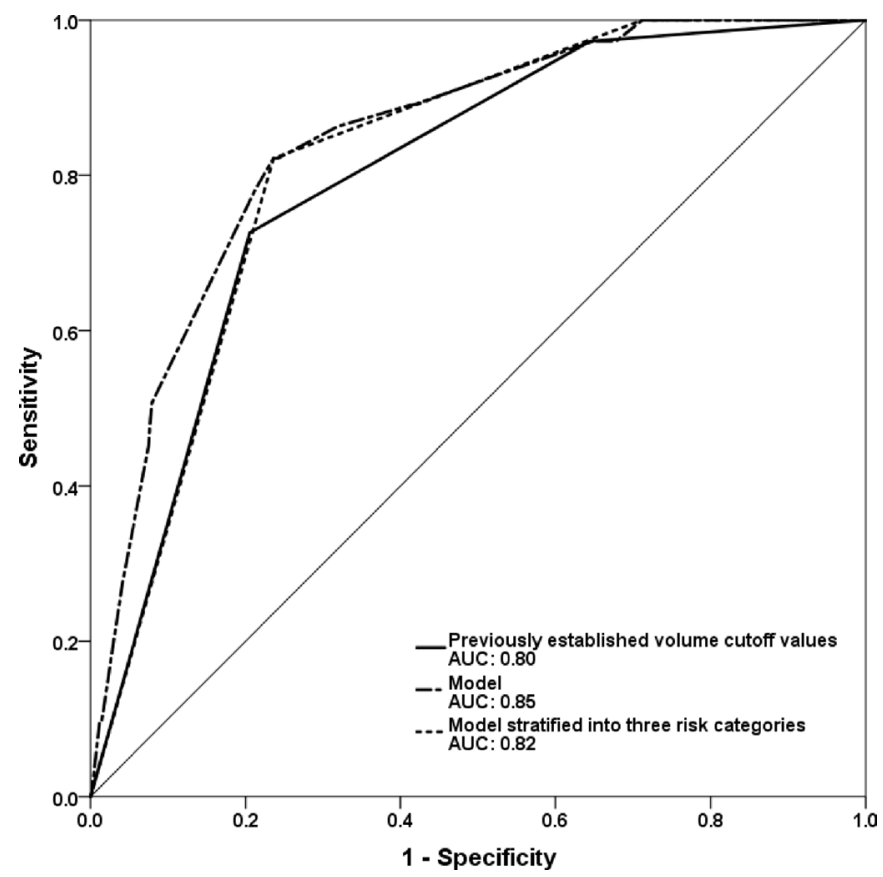

Figure 2 ROC curves of the volume cut-off values, the full model and the full model stratified into three categories for discrimination of lung cancer. AUC, area under the curve; ROC, receiver operating characteristic curve.

$30-<200 \mathrm{~mm}^{3}$ and $\left.\geq 200 \mathrm{~mm}^{3}\right),{ }^{11} 14$ only location in the right upper lung and central distribution provided incremental value, while nodule shape and margin did not improve lung cancer discrimination. This contrasts findings in baseline nodules, where aside of location, nodule morphology remained significantly associated with lung cancer when corrected for nodule size. ${ }^{19-21}$ This discrepancy may be caused by the augmented predictive information of nodule size in new solid nodules,

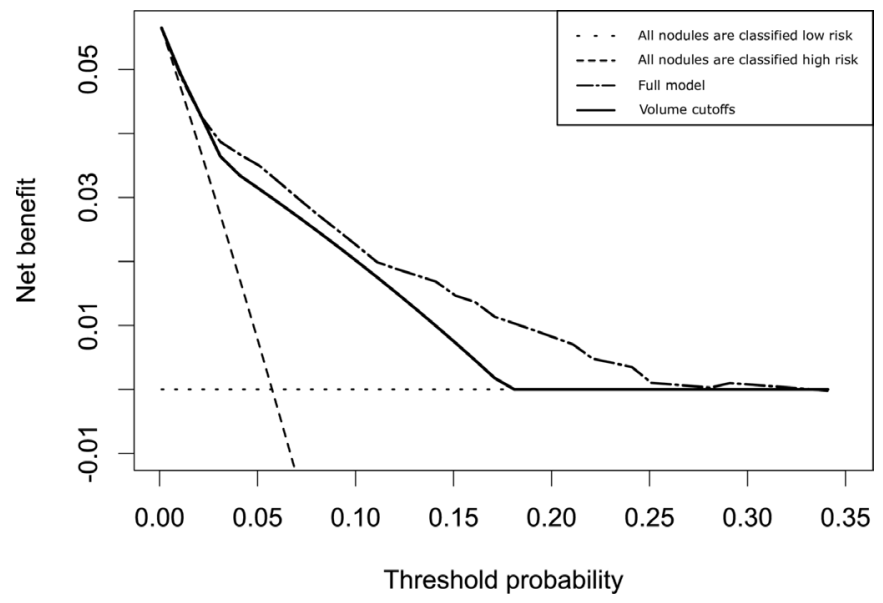

Figure 3 Decision curve analysis of the full model and volume cut-offs alone. Net benefit: sum of true-positive minus false-positive classifications weighted by the respective risk threshold. For a specific threshold probability, a larger net benefit indicates a greater number of true positive predictions without increase in the rate of false positives. Not using a model would assume that all nodules have the same risk and is illustrated by the two alternatives of either assuming that all nodules are low risk or that all nodules are high risk. This figure illustrates that, when compared with the volume cut-offs, clinical utility of the model is pronounced at higher risk thresholds. which developed in a short and known timeframe, as compared with baseline nodules, that could have been present for years before detection. The volume of a baseline nodule primarily represents its current size, whereas the volume of a new nodule more directly translates to its growth rate. This is supported by the observation that only morphological characteristics forfeit their predictive association through addition of nodule volume, while growth independent features remain significant predictors. Next to that, new solid nodules tend to be smaller than baseline nodules at initial detection, ${ }^{14}$ which could hamper classification of morphology. Fourth, visibility as very small nodule in retrospect was significantly associated with lung cancer when combined with the volume cut-offs. While this finding could have implications for new nodules $\geq 30 \mathrm{~mm}^{3}$ at detection where $22 \%(6 / 27)$ of those visible in retrospect as small nodule were lung cancer as compared with $8 \%(65 / 824)$ of those not visible in retrospect $(\mathrm{P}=0.02)$, implications for nodules $<30 \mathrm{~mm}^{3}$ seem redundant considering the respective comparison of $<1 \%(2 / 228)$ versus $0 \%(0 / 201, p=0.501)$ being lung cancer. Nodules visible in retrospect likely are persisting nodules that could explain their higher cancer risk when further growing. Fifth, the identified new solid nodule characteristics did not significantly improve risk stratification by volume when considering a three category (low risk, intermediate risk and high risk) stratification approach such as advocated in current guidelines. ${ }^{811} 14$ Although some of the selected nodule characteristics provided incremental discriminatory information and clinical utility in decision curve analysis, it was limited compared with the volume cut-offs.

This study has limitations. Nodules that remained $<15 \mathrm{~mm}^{3}$ could not be included as they were below the NELSON trial's detection limit and were not registered by radiologists. Another possible limitation may be observer variation that was not considered. However, single read was only performed by radiologists with at least 6 years of experience in thoracic imaging. Next, only solid nodules were included, with exclusion of partsolid and pure ground glass nodules. Furthermore, nodules without an additional follow-up scan within the NELSON trial were excluded from the analysis to provide the most consistent appreciation of benign and malignant nodules. Because these nodules likely were benign, the proportion of lung cancers may be slightly overrepresented. The overall occurrence and lung cancer probability of new solid nodules within the NELSON trial were reported before. ${ }^{14}$

In new solid nodules detected during incidence screening rounds of LDCT lung cancer screening, morphological and location characteristics only have limited incremental discriminatory value for lung cancer additional to volume cut-offs. Nodule characteristics not influenced by nodule growth, such as location in the right upper lung and a central distribution, can potentially improve volume-based risk stratification, but in a three category (low, intermediate and high risk) stratification approach, this is negligible. Newly detected nodules $\geq 30 \mathrm{~mm}^{3}$ and visible as small nodule $\left(<15 \mathrm{~mm}^{3}\right)$ in retrospect have a high lung cancer probability. Overall, new solid nodule volume and, therefore, speed of growth is the strongest predictor for lung cancer.

Contributors JEW, MAH, RV and MO were involved in the conception, hypotheses delineation and design of the study. All authors acquired the data or analysed and interpreted the data. All authors wrote the article or were substantially involved in its revision before submission.

Funding The NELSON-trial was sponsored by: Netherlands Organisation for Health Research and Development (ZonMw); Dutch Cancer Society Koningin Wilhelmina Fonds (KWF); Stichting Centraal Fonds Reserves van Voormalig Vrijwillige Ziekenfondsverzekeringen (RvvZ); Siemens Germany; Rotterdam Oncologic Thoracic 
Steering committee (ROTS); G. Ph. Verhagen Trust; Flemish League Against Cancer; Foundation Against Cancer; and Erasmus Trust Fund.

Disclaimer The funders had no role in study design, data collection and analysis, decision to publish or preparation of the manuscript.

Competing interests CMvdA reports grants from Symposium Thoracic Oncology, grants from American Thoracic Society, grants from Lancet Respiratory Medicine, outside the submitted work. KN reports grants from Flemish League against Cancer, grants from the Belgian Foundation against Cancer, during the conduct of the study. HJMG reports grants from Eli Lilly, Roche, MSD, BMS and Novartis, outside the submitted work. HJdK took part in a 1-day advisory meeting on biomarkers organised by M.D. Anderson/Health Sciences during the 16th World Conference on Lung Cancer, outside the submitted work.

Patient consent Not required.

Ethics approval The NELSON trial (trial registration number, ISRCTN63545820) was approved by Ethics Committees of all participating centres in the Netherlands and Belgium.

Provenance and peer review Not commissioned; externally peer reviewed.

(c) Article author(s) (or their employer(s) unless otherwise stated in the text of the article) 2018. All rights reserved. No commercial use is permitted unless otherwise expressly granted.

\section{REFERENCES}

1 Siegel RL, Miller KD, Jemal A, et al. Cancer statistics, 2017. CA Cancer J Clin 2017:67:7-30.

2 van der Aalst CM, Ten Haaf K, de Koning HJ. Lung cancer screening: latest developments and unanswered questions. Lancet Respir Med 2016:4:749-61.

3 Smith RA, Andrews K, Brooks D, et al. Cancer screening in the United States, 2016: A review of current American Cancer Society guidelines and current issues in cancer screening. CA Cancer J Clin 2016:66:95-114.

4 Moyer VA. Screening for lung cancer: U.S. Preventive Services Task Force recommendation statement. Ann Intern Med 2014;160:330-338-8.

5 de Koning HJ, Meza R, Plevritis SK, et al. Benefits and harms of computed tomography lung cancer screening strategies: A comparative modeling study for the U.S. Preventive services task force. Ann Intern Med 2014;160:311-20.

6 American College of Radiology. LungRADS ${ }^{\mathrm{TM}}$ Version 1.0 Assessment Categories Release date: April 28, 2014. https://www.acr.org/-/media/ACR/Files/RADS/Lung RADS/LungRADS_AssessmentCategories.pdf?la=en (accessed Apr 2016).

$7 \mathrm{Xu} \mathrm{DM}$, Gietema $\mathrm{H}$, de Koning $\mathrm{H}$, et al. Nodule management protocol of the NELSON randomised lung cancer screening trial. Lung Cancer 2006;54:177-84.

8 Callister MEJ, Baldwin DR, Akram AR, et al. British Thoracic Society guidelines for the investigation and management of pulmonary nodules: accredited by NICE. Thorax 2015;70:ii1-ii54.

9 MacMahon H, Austin JH, Gamsu G, et al. Guidelines for management of small pulmonary nodules detected on CT scans: a statement from the Fleischner Society. Radiology 2005;237:395-400.

10 MacMahon H, Naidich DP, Goo JM, et al. Guidelines for management of incidental pulmonary nodules detected on CT images: from the fleischner society 2017 Radiology 2017;284:161659-243.

11 Oudkerk M, Devaraj A, Vliegenthart R, et al. European position statement on lung cancer screening. Lancet Oncol 2017;18:e754-e766.

12 Henschke Cl, Yankelevitz DF, Naidich DP, et al. CT screening for lung cancer: suspiciousness of nodules according to size on baseline scans. Radiology 2004:231:164-8.
13 Horeweg N, van Rosmalen J, Heuvelmans MA, et al. Lung cancer probability in patients with CT-detected pulmonary nodules: a prespecified analysis of data from the NELSON trial of low-dose CT screening. Lancet Oncol 2014;15:1332-41.

14 Walter JE, Heuvelmans MA, de Jong PA, et al. Occurrence and lung cancer probability of new solid nodules at incidence screening with low-dose CT: analysis of data from the randomised, controlled NELSON trial. Lancet Oncol 2016;17:907-16.

15 Walter JE, Heuvelmans MA, Oudkerk M. Small pulmonary nodules in baseline and incidence screening rounds of low-dose CT lung cancer screening. Trans/ Lung Cancer Res 2017:6:42-51.

16 Pinsky PF, Gierada DS, Nath PH, et al. Lung Cancer Risk Associated With New Solid Nodules in the National Lung Screening Trial. Am J Roentgenol 2017;209:1009-14.

17 Schabath MB, Massion PP, Thompson ZJ, et al. Differences in patient outcomes of prevalence, interval, and screen-detected lung cancers in the CT arm of the national lung screening trial. PLoS One 2016;11:e0159880.

18 Heuvelmans MA, Oudkerk M, de Jong PA, et al. The impact of radiologists' expertise on screen results decisions in a CT lung cancer screening trial. Eur Radiol 2015;25:792-9.

19 Xu DM, van Klaveren RJ, de Bock GH, et al. Limited value of shape, margin and CT density in the discrimination between benign and malignant screen detected solid pulmonary nodules of the NELSON trial. Eur J Radiol 2008;68:347-52.

20 McWilliams A, Tammemagi MC, Mayo JR, et al. Probability of cancer in pulmonary nodules detected on first screening CT. N Engl J Med Overseas Ed 2013;369:910-9.

21 Xu DM, van der Zaag-Loonen HJ, Oudkerk M, et al. Smooth or attached solid indeterminate nodules detected at baseline CT screening in the NELSON study: cancer risk during 1 year of follow-up. Radiology 2009;250:264-72.

22 Horeweg N, van der Aalst CM, Thunnissen E, et al. Characteristics of lung cancers detected by computer tomography screening in the randomized NELSON trial. Am J Respir Crit Care Med 2013;187:848-54.

23 van lersel CA, de Koning HJ, Draisma G, et al. Risk-based selection from the general population in a screening trial: Selection criteria, recruitment and power for the Dutch-Belgian randomised lung cancer multi-slice CT screening trial (NELSON). Int J Cancer 2007;120:868-74.

24 van Klaveren RJ, Oudkerk M, Prokop M, et al. Management of lung nodules detected by volume CT scanning. N Engl J Med Overseas Ed 2009;361:2221-9.

25 Yousaf-Khan $U$, van der Aalst $C$, de Jong PA, et al. Final screening round of the NELSON lung cancer screening trial: the effect of a 2.5-year screening interval. Thorax 2017;72:48-56.

26 Gurney JW. Determining the likelihood of malignancy in solitary pulmonary nodules with Bayesian analysis. Part I. Theory. Radiology 1993;186:405-13.

27 Zhao YR, Heuvelmans MA, Dorrius MD, et al. Features of resolving and nonresolving indeterminate pulmonary nodules at follow-up CT: The NELSON Study. Radiology 2014:270:872-9.

28 Yousaf-Khan U, van der Aalst C, de Jong PA, et al. Risk stratification based on screening history: the NELSON lung cancer screening study. Thorax 2017;72:819-24.

29 DeLong ER, DeLong DM, Clarke-Pearson DL. Comparing the areas under two or more correlated receiver operating characteristic curves: a nonparametric approach. Biometrics 1988;44:837-45

30 Vickers AJ, Cronin AM, Elkin EB, et al. Extensions to decision curve analysis, a novel method for evaluating diagnostic tests, prediction models and molecular markers. BMC Med Inform Decis Mak 2008;8:53.

31 Steyerberg EW, Vergouwe Y. Towards better clinical prediction models: seven steps for development and an ABCD for validation. Eur Heart J 2014;35:1925-31.

32 Bach PB, Mirkin JN, Oliver TK, et al. Benefits and Harms of CT Screening for Lung Cancer. JAMA 2012:307:2418. 